\title{
Interactive comment on "Plant assemblages in atmospheric deposition" by Ke Dong et al.
}

\section{Anonymous Referee \#2}

\section{Received and published: 9 July 2019}

\section{General Comments:}

In the paper the authors examine via genetic techniques the deposition flux of plant derived material in Korea. The topic is relevant and interesting, especially considering the potential climatic interactions of primary biogenic aerosols (PBAPs). Any further insight on PBAPs emissions and deposition is for sure a much needed information. The paper is well written and clear, however the reviewer would like some more clarification about the sampling strategy used to compute deposition fluxes and deposition velocities in the paper.

Specific Comments:

Page 3, Lines 5-7: How did the building height compares with the forested area around? Was the sampler located significantly above the treetops? A figure showing the samplers and the sampling location would greatly help 
Page 3, Lines 10-11: These lines implies that both deposition and concentration samples were taken monthly. Was the Andersen sampler operated continuously for the month? Were there any issue in saturation of the substrates due to overcollection?

Page 3, Line 12: What are the specifications of such custom-made sampler? The geometry of the collector do impact the deposition process, so how was this custom made sampler validated? In the reviewer's view these are needed information that are lacking also in the referenced Woo et al., 2018 and Han et al., 2016 papers (referred in Woo et al., 2018 regarding the custom made sampler).

Page 3, Line 13: How far were the deposition sampler and the Andersen one? If they were co-located too close to each other, the active air sampling of the Andersen could affect the deposition on the custom made sampler. Again a figure of the sampling setup would greatly help instead of referring to Woo et al., 2018 (in which the figure of the sampler is in the supplementary materials).

Page 4, Lines 11-12: There is a mismatch in the units for flux and concentration. The flux is stated to be reported in $\mathrm{CN} \mathrm{cm}-2$ month-1, but the concentration is stated to be measured in $\mathrm{CN}$ m-3.

Page 6, Lines 8-10: That seems an extremely anomalous result, which is not further discussed. How do the authors explain that? Was the rain sampler from which precipitation data are taken sufficiently close to the deposition experiments or was it far away enough to justify local differences in rainfall amounts?

Page 6, Lines 27-28: Deposition velocities are computed as the ratio between the deposition sampler and the Andersen one. Given simultaneous measurement of the two it is reasonable to expect that, at least for dry deposition, the mass collected on one sampler strongly correlates with the mass collected on the other one (Mohan, 2016). A "decoupling" between the samplers could also explain some issues in computed deposition velocities, were some kind of mass-comparison tests performed on the samplers? 
Page 8, Lines 28-30: The reviewer does not really agree, there's no information in this study to support the actual existence of a taxon-dependent rainout for the sampled ACPD pollens, nor to support a prevalence of washout over rainout. The lack of differences between wet and dry deposition samples' structures might also be simply due the -lackof any taxon-dependence to rainout, rather than the more complicated assumption of washout prevalence over taxon-dependent rainout processes. The reviewer suggests rephrasing.

Page 9, Lines 28-29: Again this is a speculation (see previous comment). The reviewer suggests rephrasing.

Cited References: Mohan S. M. (2016) "An overview of particulate dry deposition: measuring methods, deposition velocity and controlling factors", Int. J. Environ. Sci. Technol., 13:387-402.

Interactive comment on Atmos. Chem. Phys. Discuss., https://doi.org/10.5194/acp-2019-487, 2019. 